IHES-P /00/xx

\title{
Characterizing Volume Forms
}

\author{
Pierre Cartier \\ Ecole Normale Supérieure \\ 45 rue d'Ulm F-75005 Paris \\ Marcus Berg, Cécile DeWitt-Morette and Alex Wurm \\ Department of Physics and Center for Relativity, \\ University of Texas, Austin, TX 78712
}

\begin{abstract}
Old and new results for characterizing volume forms in functional integration.
\end{abstract}




\section{The Wiener measure}

Defining volume forms on infinite dimensional spaces is a key problem in the theory of functional integration. The first volume form used in functional integration has been the Wiener measure. From the several equivalent definitions of the Wiener measure, we choose one [1] which can easily be extended for use in Feynman integrals. We recall the CameronMartin and Malliavin formulae because they are, respectively, integrated and infinitesimal formulae for changes of variable of integration which can be imposed on volume forms other than the Wiener measure.

\subsection{Definition}

The Wiener measure $\gamma$ on the space $\mathbb{W}$ of pointed continuous paths $w$ on the time interval $T=[0,1]$

$$
w:[0,1] \longrightarrow \mathbb{R}, \quad w(0)=0,
$$

can be characterized by the equation

$$
\int_{\mathbb{W}} d \gamma(w) \exp \left(-i\left\langle w^{\prime}, w\right\rangle\right)=\exp \left(-\frac{1}{2} \int_{T} \int_{T} d w^{\prime}(t) d w^{\prime}\left(t^{\prime}\right) \inf \left(t, t^{\prime}\right)\right)
$$

where $w^{\prime}$ is an element of the topological dual $\mathbb{W}^{\prime}$ of $\mathbb{W}$, i.e. a bounded measure on the semi-open interval $T=] 0,1]$

$$
\left\langle w^{\prime}, w\right\rangle=\int_{T} d w^{\prime}(t) w(t)
$$

\subsection{Cameron-Martin formula}

The Cameron-Martin formula can be written

$$
\int_{\mathbb{W}} d \gamma(w) F(w+\varphi)=\int_{\mathbb{W}} d \gamma(w) J(\varphi, w) F(w)
$$

where $J(\varphi, w)$ is the Radon-Nikodym derivative

$$
J(\varphi, w)=\frac{d \gamma(w-\varphi)}{d \gamma(w)} .
$$

This formally obvious expression for $J(\varphi, w)$ has a not-so-obvious explicit expression; when $\varphi \in L^{2,1}$, i.e. when $\dot{\varphi}(t)$ is square integrable, and $\varphi(0)=0$, then円

$$
J(\varphi, w)=\exp \left(-\frac{1}{2} \int_{T} d t \dot{\varphi}(t)^{2}+\int_{T} d w(t) \dot{\varphi}(t)\right) .
$$

\footnotetext{
${ }^{1}$ The boundary condition $\varphi(0)=0$ is required in order that $w+\varphi$ belong to $\mathbb{W}$ when $w$ does, since $w(0)=0$ for every element $w$ of $\mathbb{W}$.
} 
The meaning of the second term is subtle since $w$ is not of bounded variation. If $\varphi \in C^{2}(0,1)$ with the boundary conditions $\varphi(0)=\dot{\varphi}(1)=0$, the second term can be integrated by parts — giving $-\int_{T} d t w(t) \ddot{\varphi}(t)$ - and then can be extended by continuity in the $L^{2,1}$ norm for $\varphi$.

A heuristic proof of the Cameron-Martin formula makes the explicit expression (5) "obvious". Let us write formally

$$
d \gamma(w)=\mathcal{D} w \exp (-\pi Q(w))
$$

where $\mathcal{D}$ is a translation-invariant symbol $\mathbb{f}^{\mathbb{f}}$

$$
\mathcal{D}(w+\varphi)=\mathcal{D} w
$$

and where $Q$ follows from the definition (1) of the Wiener measure:

$$
\int_{\mathbb{W}} \mathcal{D} w \exp (-\pi Q(w)) \exp \left(-2 \pi i\left\langle w^{\prime}, w\right\rangle\right)=\exp \left(-\pi W\left(w^{\prime}\right)\right)
$$

with

$$
W\left(w^{\prime}\right)=2 \pi \int_{T} \int_{T} d w^{\prime}(t) d w^{\prime}\left(t^{\prime}\right) \inf \left(t, t^{\prime}\right) .
$$

By analogy with the finite dimensional case (39), the quadratic form $Q$ on $\mathbb{W}$ is required to be the inverse of $W$ on $\mathbb{W}^{\prime}$ in the following sense. Represent $W$ and $Q$ as

$$
W\left(w^{\prime}\right)=\left\langle w^{\prime}, G w^{\prime}\right\rangle \quad \text { and } \quad Q(w)=\langle D w, w\rangle ;
$$

then $Q$ is said to be the inverse of $W$ if

$$
D G=\mathbb{I} .
$$

It follows from (9) and (10) that

$$
G w^{\prime}(t)=2 \pi \int_{T} d w^{\prime}\left(t^{\prime}\right) \inf \left(t, t^{\prime}\right) .
$$

It follows from (11) and (10) that

$$
Q(w)=\frac{1}{2 \pi} \int_{T} d t\left(\frac{d w(t)}{d t}\right)^{2}=\frac{1}{2 \pi} \int_{T} \frac{(d w(t))^{2}}{d t} .
$$

\footnotetext{
${ }^{2}$ The symbol $\mathcal{D}$ is often used in physics where $\mathcal{D} w:=\prod_{t} d w(t)$. Here it is defined by (8), which in finite dimensions reduces to (41).
} 
The Cameron-Martin formula (3) is now the obvious statement

$$
\int_{\mathbb{W}} \mathcal{D} w \exp (-\pi Q(w)) F(w+\varphi)=\int_{\mathbb{W}} \mathcal{D} w \exp (-\pi Q(w-\varphi)) F(w)
$$

that is $J(\varphi, w)=\exp \pi(Q(w)-Q(w-\varphi))$. We calculate

$$
\begin{aligned}
Q(w-\varphi) & =\frac{1}{2 \pi} \int_{T} d t\left(\frac{d}{d t}(w-\varphi)(t)\right)^{2} \\
& =\frac{1}{2 \pi}\left(Q(w)+\int_{T} d t \dot{\varphi}(t)^{2}-2 \int_{T} d w(t) \dot{\varphi}(t)\right)
\end{aligned}
$$

formula (5) follows immediately and this completes the heuristic demonstration.

\subsection{An analogy with the Dobrushin-Lanford-Ruelle characteriza- tion of Gibbs states}

What is missing in the heuristic proof of the Cameron-Martin formula to be rigorous? The difficulty is that the Brownian trajectories $w$ are so rough that $Q(w)$ is infinite if calculated as the limit of the Riemann sums $\sum_{i=1}^{N}\left(\Delta w_{i}\right)^{2} / \Delta t_{i}$ where

$$
0=t_{0}<t_{1}<\ldots<t_{N}=1, \quad \Delta t_{i}=t_{i}-t_{i-1} \quad \text { and } \quad \Delta w_{i}=w\left(t_{i}\right)-w\left(t_{i-1}\right) .
$$

However, in the Cameron-Martin formula we need only the difference $Q(w)-Q(w-\varphi)$. The infinite part drops out in the difference provided $\varphi$ is smooth enough; e.g. if $\varphi$ is of class $C^{2}$ on $T=[0,1]$.

A similar situation occurs in statistical mechanics in the case of infinite volumes. For a configuration $w$, the formal Hamiltonian $H(w)$ may be infinite. But if a configuration $w^{\prime}$ is obtained by a local modification of $w$ - by changing the states in finitely many sites then $H(w)-H\left(w^{\prime}\right)$ is finite. This is the strategy underlying the Dobrushin-Lanford-Ruelle characterization of Gibbs states. [8]

\subsection{Malliavin formula}

According to the Malliavin formula,

$$
\int_{\mathbb{W}} d \gamma(w) D_{\varphi} F(w)=\int_{\mathbb{W}} d \gamma(w) A_{\varphi}(w) F(w)
$$

where $D_{\varphi} F$ is the Gateaux differential of $F$ in the $\varphi$-direction,

$$
D_{\varphi} F(w)=\lim _{\epsilon=0} \frac{1}{\epsilon}(F(w+\epsilon \varphi)-F(w))=\int_{T} d t \frac{\delta F(w)}{\delta w(t)} \varphi(t),
$$


and where

$$
\begin{aligned}
A_{\varphi}(w) & :=\int_{T} d w(t) \dot{\varphi}(t) \\
& =-\int_{T} d t w(t) \ddot{\varphi}(t)
\end{aligned}
$$

for $\varphi \in C^{2}(0,1)$, with $\varphi(0)=\dot{\varphi}(1)=0$. Again, the Malliavin formula is "obvious" if we use the formal expression (6) and integrate the left hand side of (16) by parts,

$$
\int_{\mathbb{W}} \mathcal{D} w \exp (-\pi Q(w)) D_{\varphi} F(w)=-\int_{\mathbb{W}} \mathcal{D} w D_{\varphi}(\exp (-\pi Q(w))) F(w) .
$$

The Cameron-Martin formula and its infinitesimal form, the Malliavin formula, pave the way for defining a formal translation-invariant symbol " $\mathcal{D}$ ". In this paper, we propose an infinitesimal characterization of $\mathcal{D}$; namely, given an arbitrary functional $U$ integrable by $\mathcal{D} w$, the translation invariance of $\mathcal{D}$ can be expressed by integrating by parts

$$
\int_{\mathbb{W}} \mathcal{D} w \frac{\delta U}{\delta w(t)}=-\int_{\mathbb{W}} \frac{\delta}{\delta w(t)} \mathcal{D} w \cdot U=0 \quad \forall U
$$

\subsection{Some lessons from the Malliavin formula}

- Let us write formally

$$
d \gamma(w)=\mu(w) \mathcal{D} w
$$

where $\mu(w)$, often called "measure" in physics, is not necessarily $\exp (-\pi Q(w))$.

Malliavin's formula reads

$$
\int_{\mathbb{W}} \mu(w) \mathcal{D} w\left(D_{\varphi} F(w)-A_{\varphi}(w) F(w)\right)=0
$$

which, by a formal integration by parts, becomes

$$
\int_{\mathbb{W}} \mathcal{D} w\left(D_{\varphi} \mu(w)+A_{\varphi}(w) \mu(w)\right) F(w)=0 .
$$

Since $F(w)$ is arbitrary, Malliavin's formula is equivalent to

$$
D_{\varphi} \mu(w)+A_{\varphi}(w) \mu(w)=0
$$

for all $\varphi$ sufficiently regular, e.g. $\varphi \in C^{2}(0,1)$. The "measure" $\mu(w)$ is, modulo a multiplicative constant, characterized by (24). If $\mu(w)=\exp (-\pi Q(w))$ as before, then

$$
D_{\varphi} Q(w)=\frac{1}{\pi} A_{\varphi}(w)
$$


According to (18), $A_{\varphi}(w)$ is bilinear in $\varphi$ and $w$; hence $Q$ is quadratic in $w$ and we recover formula (13).

- The above remark is a heuristic proof that the Wiener measure $\gamma$ is characterized by Malliavin's formula. The proof can be made rigorous by choosing in (16)

$$
F(w)=\exp \left(-2 \pi i\left\langle w^{\prime}, w\right\rangle\right), \quad w^{\prime} \in \mathbb{W}^{\prime} .
$$

- Conversely the Cameron-Martin formula provides a rigorous proof of the Malliavin formula (16): replace $\varphi$ by $\epsilon \varphi$ in (3) and take the derivative of both sides with respect to $\epsilon$. At $\epsilon=0$, one checks that

$$
\left.\frac{d}{d \epsilon} J(\epsilon \varphi, w)\right|_{\epsilon=0}=A_{\varphi}(w) .
$$

- The Malliavin formula can be used for realizing creation and annihilation operators on bosonic Fock spaces, [2] thanks to the Wiener chaos isomorphism: Let $\mathcal{H}:=L^{2,1}(T)$ be a one-particle (real) Hilbert space with scalar product $\left(\varphi_{1} \mid \varphi_{2}\right)=\int_{T} d t \dot{\varphi}_{1}(t) \dot{\varphi}_{2}(t)$. Let $\mathcal{F}(\mathcal{H})$ be the Fock space with vacuum $\Omega$. The Wiener chaos is an isomorphism (see e.g. 9])

$$
L^{2}(\mathbb{W}, d \gamma) \simeq \mathcal{F}(\mathcal{H})
$$

With

$$
A_{\varphi}(w):=\int_{T} d w(t) \dot{\varphi}(t)
$$

one obtains

$$
\int_{\mathbb{W}} d \gamma(w) A_{\varphi_{1}}(w) A_{\varphi_{2}}(w)=\int_{T} d t \dot{\varphi}_{1}(t) \dot{\varphi}_{2}(t) .
$$

It is enough to consider the case $\varphi_{1}=\varphi_{2}=\varphi$. This fundamental formula can then be established by a linear change of variable of integration in $\mathbb{W}$,

$$
A_{\varphi}: w \longmapsto \int_{T} d w(t) \dot{\varphi}(t) .
$$

Eq. (27) says that the map from $\mathcal{H}$ to $\mathcal{F}$,

$$
\begin{aligned}
\mathcal{H}:=L^{2,1}(T) & \longrightarrow \mathcal{F}:=L^{2}(\mathbb{W}, d \gamma) \\
\text { by } \quad & \longmapsto A_{\varphi}
\end{aligned}
$$

is an isometry.

The Malliavin formula (16), with $\varphi=\varphi_{1}$ and $F=A_{\varphi_{2}}$, gives another proof of (27)

$$
\left(\varphi_{1} \mid \varphi_{2}\right)=\int_{\mathbb{W}} d \gamma(w) D_{\varphi_{1}}\left(A_{\varphi_{2}}(w)\right)=\int_{\mathbb{W}} d \gamma(w) A_{\varphi_{1}}(w) A_{\varphi_{2}}(w)
$$


and the following commutation relations are obvious

$$
\begin{aligned}
{\left[D_{\varphi_{1}}, D_{\varphi_{2}}\right] } & =0 \\
{\left[A_{\varphi_{1}}, A_{\varphi_{2}}\right] } & =0 \\
{\left[D_{\varphi_{1}}, A_{\varphi_{2}}\right] } & =\left(\varphi_{1} \mid \varphi_{2}\right) .
\end{aligned}
$$

Therefore

$$
a^{\dagger}(\varphi):=A_{\varphi}-D_{\varphi} \quad \text { and } \quad a(\varphi):=D_{\varphi}
$$

obey the bosonic commutation relation of creation and annihilation operators on $\mathcal{F}$, respectively,

$$
\left[a\left(\varphi_{1}\right), a^{\dagger}\left(\varphi_{2}\right)\right]=\left(\varphi_{1} \mid \varphi_{2}\right) \cdot \mathbb{I},
$$

other commutators vanishing. It can be proved that $a$ and $a^{\dagger}$ are adjoint in the Hilbert space $L^{2}(\mathbb{W}, d \gamma)$ by integrating by parts the Malliavin formula (16) with $F=F_{1} F_{2}$

$$
\int_{\mathbb{W}} d \gamma D_{\varphi} F_{1} \cdot F_{2}=\int_{\mathbb{W}} d \gamma F_{1} \cdot\left(A_{\varphi} F_{2}-D_{\varphi} F_{2}\right) .
$$

The vacuum $\Omega \in \mathcal{F}$ is the constant function equal to 1 . If a functional $F$ of the Brownian path $w$ acts on $\mathcal{F}$ by multiplication, i.e.

$$
F(w): \Psi(w) \longmapsto F(w) \Psi(w)
$$

then we derive the tautology

$$
\langle\Omega|F| \Omega\rangle=\int_{\mathbb{W}} d \gamma(w) F(w)
$$

The Wiener measure is therefore the spectral measure corresponding to the vacuum state $\Omega$. The vacuum is characterized by

$$
a(\varphi) \Omega=D_{\varphi} \Omega=0 \quad, \quad \forall \varphi
$$

alternatively

$$
\frac{\delta \Omega(w)}{\delta w(t)}=0 \quad \text { for every } \quad t \in T .
$$

With the notation of (31) we write

$$
0=\langle F \mid a(\varphi) \Omega\rangle=\left\langle a^{\dagger}(\varphi) F \mid \Omega\right\rangle
$$

Hence the vacuum $\Omega$ is, up to a scalar, the unique state orthogonal to all $a^{\dagger}(\varphi) F$, that is to the functions $D_{\varphi} F-A_{\varphi} \cdot F$. This gives another interpretation to Malliavin's 
formula (16).

To show that $\left\{L^{2}(\mathbb{W}, d \gamma), \Omega, a(\varphi), a^{\dagger}(\varphi)\right\}$ is indeed a model of Fock space, it remains to check that the vectors, $a^{\dagger}\left(\varphi_{1}\right) \ldots a^{\dagger}\left(\varphi_{n}\right) \Omega$, make a total system for $L^{2}(\mathbb{W}, d \gamma)-$ i.e. that the finite linear combinations of $A_{\varphi_{1}} \ldots A_{\varphi_{n}}$ are dense in $L^{2}(\mathbb{W}, d \gamma)$. The Wiener chaos follows from the general theory of Fock space. The general theory includes not only the symmetric space considered here, but also the antisymmetric Fock space which we have not yet considered.

\subsection{Feynman volume form (see refs 3,4 )}

The Fourier transform of the Wiener measure (11) or (8) suggests a characterization of the Feynman volume form by its Fourier transform. Let $s \in\{1, i\}$, then we can define $\mathcal{D} x$ by

$$
\int_{\mathbb{X}} \mathcal{D} x \exp \left(-\frac{\pi}{s} Q(x)\right) \exp \left(-2 \pi i\left\langle x^{\prime}, x\right\rangle\right)=\exp \left(-s \pi W\left(x^{\prime}\right)\right)
$$

where $\mathbb{X}$ is the space of paths $x$ and the quadratic form

$$
\begin{array}{rll}
Q(x)>0 & \text { for } & s=1 \\
\text { imaginary part of } Q(x)>0 & \text { for } & s=i .
\end{array}
$$

The case $s=1$ corresponds to the Wiener measure while the case $s=i$ corresponds to the Feynman sum over paths in quantum mechanics. Everything said before can be repeated with obvious changes, e.g. the Malliavin formula.

\section{Volume forms in quantum field theory; Schwinger's dynamical principle}

The functional integral representation of the Schwinger dynamical principle has led Bryce DeWitt to the introduction of a ubiquitous volume form in quantum field theory. According to Schwinger, the variation of the probability amplitude for a transition 〈out|in〉 is given by the variation of the action $\mathbf{S}$ of the system[:

$$
\delta\langle\text { out }| \text { in }\rangle=\frac{i}{\hbar}\langle\text { out }|\delta \mathbf{S}| \text { in }\rangle
$$

where $\mathbf{S}$ is a functional of the field operators, which are globally designated by $\boldsymbol{\varphi}$.

\footnotetext{
${ }^{3}$ We use boldface for operators on Fock space.
} 


\subsection{Evolution equations for the field operators $\varphi$}

The Schwinger variational principle gives evolution equations for the field operators $\boldsymbol{\varphi}$ different from the classical Euler-Lagrange equations

$$
\frac{\delta S}{\delta \varphi}=0 .
$$

The Schwinger-Dyson equations give the quantum evolution of polynomials of fields $F(\boldsymbol{\varphi})$ for a system with classical action $S$ by the expectation value of a time ordered operator,

$$
\left\langle\operatorname{vac}\left|T\left(\frac{i}{\hbar} \frac{\delta S}{\delta \varphi} F(\boldsymbol{\varphi})+\frac{\delta F}{\delta \varphi}\right)\right| \mathrm{vac}\right\rangle=0 .
$$

(proof of eq. (35) and some of its applications can be found e.g. in the textbook by Peskin and Schroeder [11], section 9.6).

\subsection{Functional integral solution of the Schwinger principle}

To exploit the Schwinger variational principle (33), one varies an external source $J$ added to the original action $S$. The new action is

$$
S+\langle J, \varphi\rangle
$$

and the principle (33) now reads

$$
\left.\frac{\hbar}{i} \frac{\delta}{\delta J}\langle\text { out }| \text { in }\right\rangle=\langle\text { out }|\varphi| \text { in }\rangle .
$$

Bryce DeWitt has constructed the following functional integral solution of this equation (for details, see pp. 4160-4164 and related references in [四):

$$
\langle\text { out }| \text { in }\rangle=\mathcal{N} \int_{\Phi(\text { in }, \text { out })} \mu(\varphi) \mathcal{D} \varphi \exp \left(\frac{i}{\hbar}(S(\varphi)+\langle J, \varphi\rangle)\right)
$$

where

- $\mathcal{N}$ is a normalization constant,

- the domain of integration is defined by the in and out states,

- $\mathcal{D} \varphi$ is invariant under translations, 
- $\mu(\varphi)$ is, to leading order, given by the advanced Green's function $G^{+}$:

$$
\mu(\varphi)=\left|\operatorname{sdet} G^{+}(\varphi)\right|^{-1 / 2}+\ldots
$$

where "sdet" is the superdeterminant. In (37) the advanced Green's function $G^{+}$is the unique inverse of the leading nonconstant term $S^{\prime \prime}$ of the expansion of $S$ restricted to its domain of integration $\Phi($ in, out):

$$
S\left(\varphi_{0}+\delta \boldsymbol{\varphi}\right)=S\left(\varphi_{0}\right)+\frac{1}{2} S^{\prime \prime}\left(\varphi_{0}\right) \delta \boldsymbol{\varphi} \delta \boldsymbol{\varphi}+\ldots, \quad S^{\prime}\left(\varphi_{0}\right)=0 .
$$

Equations (32) and (36) define volume forms $\mu(\varphi) \mathcal{D} \varphi$ and $\mathcal{D} x$ respectively. In both cases $\mathcal{D}$ is a translation-invariant symbol. For comparing the structure of the two volume forms we write the finite dimensional version of (32) with $s=1$ :

$$
\int_{\mathbb{R}^{D}} \mathcal{D} x \exp \left(-\pi Q_{\alpha \beta} x^{\alpha} x^{\beta}\right) \exp \left(-2 \pi i x_{\alpha}^{\prime} x^{\alpha}\right)=\exp \left(-\pi W^{\alpha \beta} x_{\alpha}^{\prime} x_{\beta}^{\prime}\right)
$$

where

$$
\begin{aligned}
Q_{\alpha \beta} W^{\beta \gamma} & =\delta_{\alpha}^{\gamma} \\
\mathcal{D} x & =\left(\operatorname{det} Q_{\alpha \beta}\right)^{1 / 2} d x^{1} \ldots d x^{D} \\
& =\left(\operatorname{det} W^{\alpha \beta}\right)^{-1 / 2} d x^{1} \ldots d x^{D}
\end{aligned}
$$

Hence $G^{+}$is to field theory what $W$ is to a Gaussian on $\mathbb{R}^{D}$, that is, the covariance matrix:

$$
W^{\lambda \mu}=2 \pi \int_{\mathbb{R}^{D}} \mathcal{D} x \exp \left(-\pi Q_{\alpha \beta} x^{\alpha} x^{\beta}\right) x^{\lambda} x^{\mu} .
$$

In (36) the term $(2 \pi i / h)\langle J, \varphi\rangle$ corresponds to $-2 \pi i\left\langle x^{\prime}, x\right\rangle$ in (32); they both mean that the 1.h.s. is a Fourier transform.

In finite dimensions it is easy to write explicitly $\mathcal{D} x$; in infinite dimensions it has meaning only in the context of the integral (32). However, this implicit definition is sufficient for computing functional integrals. [3, [t it is also easy to generalize it to cases other than Gaussians.

Using a generalized formulation of $\mathcal{D} x$, or using $\mu(\varphi) \mathcal{D} \varphi$ obtained from the solution of the 
Schwinger variational principle is a matter of choice - often dictated by context. Once $\mu(\varphi)$, or at least its leading term, is defined, the following equation

$$
\langle\text { out }|T(F(\varphi))| \text { in }\rangle=\mathcal{N} \int \mu(\varphi) \mathcal{D} \varphi F(\varphi) \exp \left(\frac{i}{\hbar} S(\varphi)\right)
$$

can be exploited in a variety of cases; i.e. in cases where $F(\varphi)$ is not $\operatorname{simply} \exp (i\langle J, \varphi\rangle / \hbar)$.

\section{Volume forms in differential geometry}

We shall use differential geometry for defining volume forms on finite dimensional Riemannian and symplectic manifolds in a formulation which paves the way for the infinite dimensional case. Knowledgeable reader for whom using the infinite limit of a finite volume element is - rightly - anathema, please bear with us. Finite dimensional volume elements are useful in the following situations.

- A rule of thumb. A statement which is independent of the dimension of the space of interest has a chance to generalize to infinite dimensional spaces; for example a Gaussian on $\mathbb{R}^{D}$ defined by (39) generalizes easily to (8).

- Infinite dimensional spaces defined by a projective system of finite dimensional spaces. This strategy was used in defining Feynman volume forms by their Fourier transforms. [6, [7]

- Differential calculus on Banach spaces, and differential geometry on Banach manifolds. They are natural generalizations of their finite dimensional counterparts. For this reason we propose a formula which defines volume elements by their Lie derivatives.

Let $\mathcal{L}_{X}$ be the Lie derivative with respect to a vector field $X$ on a $D$-dimensional manifold $M^{D}$, either a (pseudo-)Riemannian manifold $\left(M^{D}, g\right)$ or a symplectic manifold $\left(M^{2 N}, \Omega\right)$; the volume forms are, respectively,

$$
\omega_{g}(x)=\left|\operatorname{det} g_{\alpha \beta}(x)\right|^{1 / 2} d x^{1} \wedge \ldots \wedge d x^{D} \quad \text { on }\left(M^{D}, g\right)
$$

and

$$
\omega_{\Omega}(x)=\frac{1}{N !} \Omega \wedge \ldots \wedge \Omega(\mathrm{N} \text { factors }) \quad \text { on }\left(M^{2 N}, \Omega\right) .
$$

In canonical coordinates $(p, q)$,

$$
\Omega=\sum_{\alpha} d p_{\alpha} \wedge d q^{\alpha}
$$


and

$$
\omega_{\Omega}=d p_{1} \wedge d q^{1} \wedge \ldots \wedge d p_{N} \wedge d q^{N}
$$

Surprisingly $\omega_{g}$ and $\omega_{\Omega}$ satisfy equations of the same structure:

$$
\begin{aligned}
\mathcal{L}_{X} \omega_{g} & =\frac{1}{2} \operatorname{Tr}\left(g^{-1} \mathcal{L}_{X} g\right) \omega_{g} \\
\mathcal{L}_{X} \omega_{\Omega} & =\frac{1}{2} \operatorname{Tr}\left(\Omega^{-1} \mathcal{L}_{X} \Omega\right) \omega_{\Omega}
\end{aligned}
$$

Riemannian and symplectic geometry are notoriously different (see e.g. McDuff 10]) and the analogies between them are not superficial. For instance, with Riemannian geometry on the left and symplectic geometry on the right

$$
\begin{array}{cc}
\int d s & \int \Omega \\
\text { geodesics } & \text { minimal surfaces } \\
\mathcal{L}_{X} g=0 \text { defines } & \mathcal{L}_{X} \Omega=0 \text { defines } \\
\text { Killing vector fields. } & \text { Hamiltonian vector fields. }
\end{array}
$$

Killing vector fields are few, Hamiltonian vector fields are many.

\subsection{The general case}

Before proving (44), (45) we consider the more general equation

$$
\mathcal{L}_{X} \omega=D(X) \cdot \omega
$$

or its integrated formulation

$$
\int_{M}\left(\mathcal{L}_{X} F\right) \omega=-\int_{M} F \mathcal{L}_{X} \omega=-\int_{M} F D(X) \cdot \omega
$$

where $\omega$ is a top form (a $D$-dimensional form on $M^{D}$ ) and $D(X)$ is a function on $M$ depending on the vector field $X$ on $M$.

- Properties of $D(X)$ dictated by properties of $\mathcal{L}_{X}$ :

$$
\mathcal{L}_{[X, Y]}=\mathcal{L}_{X} \mathcal{L}_{Y}-\mathcal{L}_{Y} \mathcal{L}_{X} \Leftrightarrow D([X, Y])=X(D(Y))-Y(D(X))
$$

On a top form:

$$
\mathcal{L}_{f X}=f \mathcal{L}_{X}+X(f) \Leftrightarrow D(f X)=f D(X)+X(f)
$$

Proof: On a top form $\omega$, the Cartan formula $\mathcal{L}_{X}=d i_{X}+i_{X} d$ yields

$$
\mathcal{L}_{X} \omega=d i_{X} \omega
$$

and since $i_{f X} \omega=i_{X}(f \omega)$, we have $\mathcal{L}_{f X} \omega=d i_{X}(f \omega)=\mathcal{L}_{X}(f \omega)$. 
- In coordinates,

$$
\omega_{\mu}(x)=\mu(x) d x^{1} \wedge \ldots \wedge d x^{D}=\mu(x) d^{D} x .
$$

By the Leibniz rule,

$$
\mathcal{L}_{X}\left(\mu d^{D} x\right)=\mathcal{L}_{X}(\mu) d^{D} x+\mu \mathcal{L}_{X}\left(d^{D} x\right) .
$$

Because $d^{D} x$ is a top form on $M^{D}$,

$$
\mathcal{L}_{X}\left(d^{D} x\right)=d\left(i_{X} d^{D} x\right)=X^{\alpha}{ }_{, \alpha} d^{D} x .
$$

Finally, combining (51), (52) and (53),

$$
\begin{aligned}
\mathcal{L}_{X} \omega_{\mu} & =\left(X^{\alpha} \mu_{, \alpha}+\mu X^{\alpha}{ }_{, \alpha}\right) d^{D} x \\
& =D(X) \cdot \omega_{\mu}
\end{aligned}
$$

with

$$
\begin{aligned}
D(X) & =\left(X^{\alpha} \mu_{, \alpha}+\mu X_{, \alpha}^{\alpha}\right) \mu^{-1} \\
& =X_{, \alpha}^{\alpha}+X^{\alpha}(\log |\mu|)_{, \alpha} .
\end{aligned}
$$

\subsection{The Riemannian case $(M, g)$}

Let $\omega_{g}(x)=\mu(x) d^{D} x$. We shall show that the basic equation (44) is satisfied if and only if $\mu(x)=$ const $|\operatorname{det} g(x)|^{1 / 2}$. Indeed

$$
\left(\mathcal{L}_{X} g\right)_{\alpha \beta}=X^{\gamma} g_{\alpha \beta, \gamma}+g_{\gamma \beta} X^{\gamma}{ }_{, \alpha}+g_{\alpha \gamma} X^{\gamma}{ }_{, \beta}
$$

and

$$
\operatorname{Tr}\left(g^{-1} \mathcal{L}_{X} g\right)=g^{\beta \alpha} X^{\gamma} g_{\alpha \beta, \gamma}+2 X_{, \alpha}^{\alpha}
$$

and, as already computed (54),

$$
\mathcal{L}_{X}\left(\mu(x) d^{D} x\right)=\left(X^{\alpha} \mu_{, \alpha}+\mu X^{\alpha}{ }_{, \alpha}\right) d^{D} x
$$

Therefore the basic equation (44) is satisfied if, and only if

$$
\left(X^{\gamma} \mu_{, \gamma}+\mu X_{, \alpha}^{\alpha}\right) \mu^{-1}=\frac{1}{2}\left(g^{\beta \alpha} X^{\gamma} g_{\alpha \beta, \gamma}+2 X^{\alpha}{ }_{, \alpha}\right)
$$

i.e.

$$
\begin{aligned}
\frac{\mu_{, \gamma}}{\mu} & =\frac{1}{2} g^{\beta \alpha} g_{\alpha \beta, \gamma} \\
& =\frac{1}{2} \partial_{\gamma} \ln |\operatorname{det} g| \\
\mu(x) & =\text { const. }|\operatorname{det} g(x)|^{1 / 2} .
\end{aligned}
$$


The equation

$$
\mathcal{L}_{X} \omega_{g}=\frac{1}{2} \operatorname{Tr}\left(g^{-1} \mathcal{L}_{X} g\right) \omega_{g}
$$

has, up to multiplication by a constant, a unique solution

$$
\omega_{g}(x)=|\operatorname{det} g(x)|^{1 / 2} d x^{1} \wedge \ldots \wedge d x^{D} .
$$

We use the classical formula

$$
\Gamma_{\alpha \gamma}^{\alpha}=\frac{1}{2} g^{\beta \alpha} g_{\alpha \beta, \gamma}
$$

with the Christoffel symbols $\Gamma^{\alpha}{ }_{\beta \gamma}$; hence (56) says

$$
\frac{1}{2} \operatorname{Tr}\left(g^{-1} \mathcal{L}_{X} g\right)=X_{; \alpha}^{\alpha}=: \operatorname{Div}_{g}(X)
$$

with the standard definition of the covariant divergence $X_{; \alpha}^{\alpha}$ of the vector field $X$, and we can write the basic equation (44) in the form

$$
\mathcal{L}_{X} \omega_{g}=\operatorname{Div}_{g}(X) \cdot \omega_{g} .
$$

If $X$ is a Killing vector field with respect to isometries, then $\mathcal{L}_{X} g=0, \mathcal{L}_{X} \omega_{g}=0$ and $X_{\alpha ; \beta}+X_{\beta ; \alpha}=0$. Hence $X_{; \alpha}^{\alpha}=0$ and (66) is satisfied.

\subsection{The symplectic case $\left(M^{D}, \Omega\right), D=2 N$}

The symplectic form $\Omega$ on $M^{2 N}$ is a closed 2-form of rank $D=2 N$.

$$
\begin{aligned}
\Omega & =\Omega_{A B} d x^{A} \wedge d x^{B} & & \text { with } A<B, \Omega_{A B}=-\Omega_{B A}, d \Omega=0 \\
& =\frac{1}{2} \Omega_{\alpha \beta} d x^{\alpha} \wedge d x^{\beta} & & \text { no restriction on the order of } \alpha, \beta \\
& =\frac{1}{2} \Omega_{\alpha \beta}\left(d x^{\alpha} \otimes d x^{\beta}-d x^{\beta} \otimes d x^{\alpha}\right) & & \\
& =\Omega_{\alpha \beta} d x^{\alpha} \otimes d x^{\beta} & &
\end{aligned}
$$

since $\Omega_{\alpha \beta}=-\Omega_{\beta \alpha}$.

Remark: There are two different definitions of the exterior product, each with its concomitant definition of exterior derivative, e.g.

$$
\begin{aligned}
d x^{1} \wedge d x^{2} & =d x^{1} \otimes d x^{2}-d x^{2} \otimes d x^{1} \\
\tilde{d} x^{1} \tilde{\wedge} \tilde{d} x^{2} & =\frac{1}{2}\left(\tilde{d} x^{1} \otimes \tilde{d} x^{2}-\tilde{d} x^{2} \otimes \tilde{d} x^{1}\right) .
\end{aligned}
$$

With the second definition, Stokes' formula for a $p$-form $\theta$ reads $\int_{M} \tilde{d} \theta=(p+1) \int_{\partial M} \theta$; with the first one it is simply $\int_{M} d \theta=\int_{\partial M} \theta$. We choose the first definition, namely

$$
d f^{1} \wedge \ldots \wedge d f^{p}=\epsilon_{j_{1} \ldots j_{p}} d f^{j_{1}} \otimes \ldots \otimes d f^{j_{p}}
$$


and in particular

$$
d x^{1} \wedge \ldots \wedge d x^{D}=\epsilon_{j_{1} \ldots j_{D}} d x^{j_{1}} \otimes \ldots \otimes d x^{j_{D}}
$$

where $\epsilon$ is totally antisymmetric.

Since $\Omega$ is of rank $D=2 N$,

$$
\Omega^{\wedge N}:=\Omega \wedge \ldots \wedge \Omega \quad(N \text { factors })
$$

is a nonzero top form on $M^{2 N}$ and the volume element

$$
\begin{aligned}
\omega_{\Omega} & =\frac{1}{N !} \Omega^{\wedge N} \\
& =\operatorname{Pf}\left(\Omega_{\alpha \beta}\right) d^{D} x=\left|\operatorname{det} \Omega_{\alpha \beta}\right|^{1 / 2} d^{D} x .
\end{aligned}
$$

We shall show that the basic equation (45) is satisfied if and only if $\omega_{\Omega}$ is proportional to the volume form $(70)$.

Proof: We define the inverse $\Omega^{-1}$ of $\Omega$, calculate the quantity $\frac{1}{2} \operatorname{Tr}\left(\Omega^{-1} \mathcal{L}_{X} \Omega\right)$, then prove the basic formula (45).

- The symplectic form $\Omega$ defines an isomorphism from the tangent bundle $T M$ to the cotangent bundle $T^{*} M$ by

$$
\Omega: X \longmapsto i_{X} \Omega
$$

We can then define

$$
X_{\alpha}:=X^{\beta} \Omega_{\beta \alpha}
$$

The inverse $\Omega^{-1}: T^{*} M \longrightarrow T M$ is given by

$$
X^{\alpha}=X_{\beta} \Omega^{\beta \alpha}
$$

with

$$
\Omega^{\alpha \beta} \Omega_{\beta \gamma}=\delta_{\gamma}^{\alpha}
$$

Note that in strict components, i.e. with $\Omega=\Omega_{A B} d x^{A} \wedge d x^{B}$ with $A<B, X_{A}$ is not equal to $X^{B} \Omega_{B A}$.

- We compute

$$
\begin{aligned}
\left(\mathcal{L}_{X} \Omega\right)_{\alpha \beta} & =X^{\gamma} \Omega_{\alpha \beta, \gamma}+\Omega_{\gamma \beta} X_{, \alpha}^{\gamma}+\Omega_{\alpha \gamma} X_{, \beta}^{\gamma} \\
& =X_{\beta, \alpha}-X_{\alpha, \beta}
\end{aligned}
$$


using $d \Omega=0$, that is $\Omega_{\beta \gamma, \alpha}+\Omega_{\gamma \alpha, \beta}+\Omega_{\alpha \beta, \gamma}=0$; hence

$$
\left(\Omega^{-1} \mathcal{L}_{X} \Omega\right)_{\beta}^{\gamma}=\Omega^{\gamma \alpha}\left(X_{\beta, \alpha}-X_{\alpha, \beta}\right)
$$

and

$$
\frac{1}{2} \operatorname{Tr}\left(\Omega^{-1} \mathcal{L}_{X} \Omega\right)=\Omega^{\gamma \alpha} X_{\gamma, \alpha}
$$

- According to Darboux' theorem, there is a coordinate system $\left(x^{\alpha}\right)$ in which the volume form $\omega_{\Omega}=\frac{1}{N !} \Omega^{\wedge N}$ is

$$
\omega_{\Omega}=d x^{1} \wedge \ldots \wedge d x^{2 N}
$$

and $\Omega=\Omega_{\alpha \beta} d x^{\alpha} \otimes d x^{\beta}$ with constant coefficients $\Omega_{\alpha \beta}$; the inverse matrix $\Omega^{\beta \alpha}$ is also made of constants, hence $\Omega^{\beta \alpha}{ }_{, \gamma}=0$.

In these coordinates

$$
\begin{aligned}
\mathcal{L}_{X} \omega_{\Omega} & =X_{, \alpha}^{\alpha} \omega_{\Omega} \\
& =\left(X_{\beta} \Omega^{\beta \alpha}\right)_{, \alpha} \omega_{\Omega} \\
& =\left(X_{\beta, \alpha} \Omega^{\beta \alpha}+X_{\beta} \Omega^{\beta \alpha}{ }_{, \alpha}\right) \omega_{\Omega} \\
& =X_{\beta, \alpha} \Omega^{\beta \alpha} \omega_{\Omega}
\end{aligned}
$$

and we conclude by using (74).

If $X$ is a Hamiltonian vector field, then $\mathcal{L}_{X} \Omega=0$ and $\mathcal{L}_{X} \omega_{\Omega}=0$. The basic equation (45) is trivially satisfied.

\section{Conclusion}

Integration by parts is the key to the progress made in this paper for characterizing volume forms. It makes possible an infinitesimal characterization of the translation invariant symbol $\mathcal{D}$,

$$
\int \mathcal{D} \varphi \frac{\delta U}{\delta \varphi(x)}=0
$$

more powerful than its global translation (7)

$$
\mathcal{D}\left(\varphi+\varphi_{0}\right)-\mathcal{D} \varphi=0
$$

The challenges we are now considering are the following: 
- Extending to infinite dimensional spaces the divergence formulae (45) and (44).

- Clarifying the often observed relationship between the volume form and the Schrödinger equation satisfied by a functional integral.

- Developing issues mentioned briefly in this paper, in particular the Dobrushin-LanfordRuelle formula, and the annihilation/creation operators defined by the Malliavin formula.

- Deriving the transformation laws of volume elements under the Cartan development mapping between two spaces of pointed paths on different manifolds.

- Extending the method from ordinary (bosonic) integration to Berezin (fermionic) integration.

\section{Acknowledgments}

Modern means of communication do not replace face-to-face brainstorming, and a group based in Paris and Austin functions thanks to contributions to travel expenses. We thank a steadfast friend John Tate who contributes to the annual visits of Pierre Cartier to Austin, and we thank the Jane and Roland Blumberg Centennial Professorship for partial support of Cécile DeWitt-Morette's visits to Paris. M. Berg is grateful to the Swedish Foundation for International Cooperation in Research and Higher Education for financial support.

\section{References}

[1] N. Bourbaki, Intégration, Chapitre 9, Masson, Paris (1982).

[2] P. Cartier, unpublished notes.

[3] P. Cartier and C. DeWitt-Morette, "A new perspective on functional integration", J. Math. Phys. 36 (1995) 2237-2312.

[4] P. Cartier and C. DeWitt-Morette, "Functional Integration", J. Math. Phys. 41 (2000) 4154-4187.

[5] Y. Choquet-Bruhat and C. DeWitt-Morette, Analysis, Manifolds, and Physics, Part II, pp.79-81, North Holland, Amsterdam (1989). 
[6] C. Morette DeWitt, "Feynman's path integral, definition without limiting procedure", Commun. Math. Phys. 28 (1972) 47-67.

[7] C. DeWitt-Morette, A. Maheshwari, and B. Nelson, "Path integration in non-relativistic quantum mechanics", Phys. Rep. 50 (1979) 255-372.

[8] G. Gallavotti, Statistical Mechanics: A short treatise, Springer-Verlag, Berlin (1999). Includes original DLR references.

[9] S. Janson, Gaussian Hilbert Spaces, Cambridge University Press, Cambridge, UK (1997).

[10] D. McDuff, "Symplectic structures", Notices of AMS 45 (1998) 952-960.

[11] M. E. Peskin and D. V. Schroeder, An Introduction To Quantum Field Theory, AddisonWesley Publishing Co, Reading, MA (1995). 